\title{
Influence of Prior Major Bleeding on the Safety and Effectiveness of Left Atrial Appendage Closure in Non-Valvular Atrial Fibrillation Patients: A Single- Center Study
}

\section{Mingzhong Zhao}

Heart Center, Zhengzhou Ninth People's Hospital, Zhengzhou, China

Cody R. Hou

University of Minnesota Medical School, Minneapolis, MN, USA

Xiaolin Xiong

University of Cologne, the Faculty of Medicine, Cologne, Germany

\section{Felix Post}

Clinic for General Internal Medicine and Cardiology, Katholisches Klinikum Koblenz-Montabaur, Germany

\section{Nora Herold}

Clinic for General Internal Medicine and Cardiology, Katholisches Klinikum Koblenz-Monyabaur,

Germany

Jens Walsleben

Clinic for General Internal Medicine and Cardiology, Katholisches Klinikum Koblenz-Montabaur,

Germany

\section{Zhaohui Meng}

Department of Cardiology, Kunming Medical University, Kunming, China

Jiangtao Yu ( $\sim$ j.yu@kk-km.de)

Katholisches Klinikum Koblenz Montabaur gGmbH https://orcid.org/0000-0003-1249-0265

\section{Research Article}

Keywords: atrial fibrillation, left atrial appendage closure, bleeding, Stroke, thromboembolism

Posted Date: April 8th, 2021

DOl: https://doi.org/10.21203/rs.3.rs-385487/v1

License: (c) (1) This work is licensed under a Creative Commons Attribution 4.0 International License. Read Full License 


\section{Abstract}

Purpose: Left atrial appendage closure (LAAC) may present an alternative to anticoagulation in patients with non-valvular atrial fibrillation (NVAF). However, evidence regarding benefit of LAAC in patients with prior major bleeding is limited. We evaluate whether a previous bleeding factor influences the safety and effectiveness of LAAC.

Methods: A total of 377 consecutive patients scheduled for LAAC were categorized into a bleeding group $(n=137)$ and non-bleeding group $(n=240)$. The implantation success and prevalence of severe periprocedural complications, and efficacy/ safety endpoints during follow-up were investigated.

Results: The bleeding group had more patients $\geq 75$ years old $(P=0.044)$, higher $\mathrm{CHA}_{2} \mathrm{DS}_{2}-\mathrm{VASc}(\mathrm{P}=0.029)$ and HAS-BLED scores $(P=0.001)$ than the non-bleeding group. Implantation success and severe periprocedural complications were similar. During an average 2 years' follow-up, major bleeding events $(P=0.917)$, all-cause death $(P=0.313)$, co-primary efficacy events $(P=0.063)$, and the cumulative survival ratio $(P=0.828)$ were comparable. However, the incidence of thromboembolism was lower in the bleeding group $(P=0.031)$. The observed thromboembolism rate was reduced by $86.1 \%(P<0.005)$ and $43.9 \%$ $(P<0.05)$ and the observed bleeding rate was decreased by 39.0\% $(P<0.05)$ and $34.8 \%(P<0.05)$ in the bleeding and non-bleeding groups, respectively. The extent of relative risk reduction in thromboembolism was significantly higher in the bleeding group $(86.1 \%$ vs. $43.9 \%, P<0.01)$.

Conclusion: LAAC may provide a safe and effective alternative to long-term antithrombotic therapy in NVAF patients with or without prior bleeding. The efficacy of LAAC in reducing thromboembolism instead of major bleeding seems to be higher in patients with versus without prior bleeding.

\section{Introduction}

Atrial fibrillation (AF) is the most common cardiac arrhythmia, among which non-valvular atrial fibrillation (NVAF) accounts for the majority of AF cases [1]. The risk of morbidity and mortality, especially due to cardioembolic stroke, is significantly higher in AF patients than those without AF [2]. In NVAF, more than $90 \%$ of intracardiac thrombi are located inside the left atrial appendage (LAA). A large transesophageal echo study showed that when thrombosis presented in NVAF patients, almost all thrombus formation was observed in the LAA [3]. Therefore, the prevention of thromboembolic complications is very important in AF patients.

For AF patients, vitamin $\mathrm{K}$ antagonists (VKA) such as warfarin used to be the mainstay of oral anticoagulation (OAC) therapy. VKA therapy results in a trend for a reduction of the risk of thromboembolism [4]. Recently, newer non-vitamin-K antagonist oral anticoagulants (NOACs) are increasingly used for anticoagulation treatment. NOACs exhibited higher therapy persistence and were associated with a more favorable benefit-harm profile compared to warfarin [5-7]. However, oral anticoagulants present several disadvantages. Warfarin has a lower compliance for long-term application because of a narrow therapeutic window requiring regular monitoring of international normalized ratio 
(INR) and bleeding risk; NOACs still confer a high risk of major bleeding [8] and are required to adjust the dose or contraindicated in patients with impaired renal function [9]. The prevention of thromboembolic events is particularly challenging in NVAF patients with a history of major bleeding. LAA closure (LAAC) using a mechanical device was deemed as an alternative treatment to anticoagulants in prevention of stroke or systemic embolism in patients with AF. Randomized controlled trials demonstrated the safety and effectiveness of LAAC in prevention of stroke, compared with oral warfarin [10, 11]. LAAC was also noninferior to NOACs in preventing cardiovascular, neurological, and bleeding events in high-risk patients with AF [12]. However, these trials excluded patients with contraindications to long-term prescription of warfarin. Data regarding the impact of prior major bleeding on thromboembolic and recurrent bleeding risks in patients with AF undergoing LAAC is scarce. The aim of the study was to investigate the effect of a history of previous major bleeding on the safety and efficacy of LAAC in patients with NVAF.

\section{Methods}

\section{Study population}

Between March 2012 to October 2018, all consecutive patients with NVAF and high bleeding risk for oral anticoagulation therapy who underwent percutaneous LAAC procedures using the WATCHMAN ${ }^{\text {TM }}(\mathrm{WM})$ occluder (Boston Scientific, Marlborough, MA, USA) at Helmut-G.-Walther-Klinikum, Lichtenfels, Germany were enrolled in the study and analyzed retrospectively. The study protocol was approved by the institutional review board. This study compiles with the Declaration of Helsinki and informed consent was obtained from each participant.

Inclusion criteria were patients with NVAF and high risk for stroke or systemic embolism $\left[\mathrm{CHA}_{2} \mathrm{DS}_{2} \mathrm{VASc}\right.$ (Congestive heart failure, Hypertension, Age $\geq 75$ years, Diabetes mellitus, Stroke / transient ischemic attack, Vascular disease, Age 65 to 74 years, Sex category) scores $\geq 2$ or presence of a prior history of stroke, transient ischemic attacks (TIA), or peripheral embolism], or with high bleeding risk [HAS-BLED (Hypertension, Abnormal renal / liver function, Stroke, Bleeding history or predisposition, Labile INR, Elderly, Drugs / alcohol concomitantly) scores $\geq 3$ or positive history of intracranial hemorrhage or gastrointestinal bleeding or other major bleeding, as well as unstable INR], or OAC refusal. Patients with severe multiple organ failure, malignancy, life expectancy shorter than one year, and refusal to provide written informed consent were not enrolled in the study. The participants were divided into two groups: bleeding group and non-bleeding group, based on presence or absence of a history of previous major bleeding. The prior major bleeding included severe bleeding events during anticoagulant treatment or a history of previous hemorrhagic disorders. Bleeding events were defined according to standardized bleeding endpoint definitions by the Bleeding Academic Research Consortium (BARC) [13]. The clinical characteristics, peri-procedural severe complications, and major adverse events at follow up were recorded and analyzed for all participants. Figure 1 illustrates a flow diagram of the patient inclusion, management, and follow up.

\section{LAAC procedure}


The implantation procedure was performed in all subjects. The LAAC procedure was similar to that reported in previous literature $[14,15]$. Briefly, this procedure was performed under general anesthesia with the guidance of fluoroscopy and transesophageal echocardiography (TEE). Intravenous heparin was administered at a dose of 70-100 IU/ $\mathrm{kg}$ to maintain an activated clotting time of 250-300 seconds after successfully puncturing the interatrial septum. All device implantation met PASS criteria (position, anchor, size, and seal) before release of the device. The successful implantation of the device was defined as complete occlusion of left atrial appendage or closure with less than $5 \mathrm{~mm}$ peri-device flow. Patients stayed in the hospital overnight and were discharged within 1 or 2 days post-procedure if no severe pericardial effusion, cardiac tamponade, significant procedure-associated bleeding, or other procedure complications were found.

\section{Patient follow-up}

The baseline characteristics, peri-procedural complications, and major adverse events during long-term follow-up in all patients were recorded. The clinical follow up visits were scheduled at 45 days, 6 months post procedure, and at the end of the study. TEE follow up was performed at least two times at 45 days and at 6 months post device implant. Post-implantation antithrombotic regimen was individually tailored based on risk factors of stroke and bleeding events at the physician's discretion. Generally, for the first 45 days after procedure, postoperative antithrombotic treatment was a combination of aspirin and warfarin or NOAC. Subjects with contraindications to warfarin were treated with a combined enoxaparin with aspirin or aspirin plus clopidogrel (dual-antiplatelet therapy). Patients switched to dual-antiplatelet therapy, which was taken until 6 months post procedure if TEE showed complete closure of LAA or no significant residual peri-device leakage (flow $<5 \mathrm{~mm}$ in width) and no evidence of device-related thrombus (DRT) at the 45 day TEE visit. Single antiplatelet treatment with aspirin was prescribed indefinitely in patients with successful closure and no DRT at the 6 month TEE visit. If TEE indicated that atrial thrombi or DRT were presented, anticoagulation regimen was restarted until complete disappearance of thrombus by repeated TEE evaluation.

\section{Endpoints of the study}

The primary endpoints of the study included: (1) implantation success rate, (2) severe peri-procedural complications within 7 days, and (3) primary efficacy endpoints and primary safety endpoints during long-term follow up. Severe peri-procedural complications were defined as: ischemic stroke, transient ischemic attack (TIA), systemic embolism, major bleeding events (intracranial hemorrhage, gastrointestinal bleeding, and other major bleedings), pericardial effusion, cardiac tamponade, severe vascular complication and device-related death. Primary efficacy endpoints were defined as composite thromboembolic events (ischemic stroke / TIA / systemic embolism), all-cause death events [cardiovascular (CV) / unexplained death, non-CV / unexplained death], and combined primary (Coprimary) efficacy events (composite thromboembolic events / all-cause death). The primary safety endpoints were the occurrences of severe adverse events over the course of long-term follow up, including major bleeding and DRT events. 


\section{Statistical analysis}

Continuous variables are displayed as mean \pm SEM or median and interquartile range (IQR), based on the normality of the distributions as evaluated by graphical study and Shapiro-Wilk tests. Categorical variables are described as count and percentage. Continuous variables were compared using Student $t$ tests or Mann-Whitney

Wilcoxon U tests, depending on the normality of distributions. For categorical variables, Chi-Square or Fisher's exact test was used to assess the differences between groups. Comparison of event rate was performed by Cox proportional hazard model with the estimation of hazard ratios (HR) and their 95\% confidence intervals $(\mathrm{Cl})$ among groups. Adjustment for potential confounding factors was conducted with propensity score-matching (PSM) analysis. Kaplan-Meier curves were used for graphical assessment of time dependent events, and the differences between groups were evaluated by Log-rank test.

The efficacy of LAAC for preventing thromboembolic events and decreasing bleeding risk was evaluated by comparing the observed annual event rate and the predicted annual risk using the Chi-Square test. The expected annual rates of thromboembolic and bleeding events were estimated according to the corresponding incidence rates based on $\mathrm{CHA}_{2} \mathrm{DS}_{2}$-VASc score and HAS-BLED score, respectively[16,17]. The expected annual rates of thromboembolic and bleeding events in a group were calculated as the quotients of the total expected annual thromboembolic and bleeding rates divided by total numbers of patients, respectively. The actual annual rates of thromboembolic and bleeding events were calculated as follows: the total numbers of thromboembolic or bleeding events during follow-up were divided by the total patient-years of follow-up and were multiplied by 100 to get the actual annual rate of thromboembolic or bleeding events, which was expressed as thromboembolic or bleeding events per 100 patient-years, respectively. Differences in the efficacy of LAAC in reducing thromboembolic and major bleeding risks between the two groups were compared with Chi-Square tests. A P-value $<0.05$ was considered significant. Analyses were performed using SPSS version 22.0 (SPSS Inc., Chicago, Illinois).

\section{Results}

\section{Baseline clinical characteristics}

Out of 387 consecutive patients with NVAF who underwent LAAC, six patients were switched to the Amplatzer Cardiac Plug device (St. Jude Medical, Golden Valley, MN) because of unsuitable LAA anatomy for WATCHMAN ${ }^{\top M}$ (WM) device. Four cases of implantation with WM device were halted due to 3 cases of significant pericardial effusion / cardiac tamponade and 1 case of repeated device-associated thrombus. 377 (97.4\%) cases were successfully implanted with WM occluders, including 137 cases in the bleeding group and 240 cases in the non-bleeding group. In the bleeding group, prior major bleedings were intracranial in 20 patients $(14.6 \%)$, gastrointestinal in 86 patients $(62.8 \%)$, and other major bleeding in 31 patients (22.6\%). No significant difference in procedural success rate of WATCHMAN ${ }^{\mathrm{TM}}$ occluders was found between the two groups ( $97.6 \%$ vs $96.8 \%, P=0.156)$. 
Table 1 shows the baseline demographic and clinical characteristics of the study population. The bleeding group more often had individuals $\geq 75$ years old and had significantly higher mean $\mathrm{CHA}_{2} \mathrm{DS}_{2}{ }^{-}$ VASc and HAS-BLED scores, but was less likely to take oral warfarin compared to the non-bleeding group. The remaining clinical variables were comparable between the two groups (Table 1).

\section{Peri-procedure complications}

The total adverse events over the peri-procedure period (i.e. within 7 days of the index procedure) occurred in 11 cases (2.9\%). Stroke, major bleeding, pericardial effusion/ cardiac tamponade, and severe vascular complication occurred in $0.3 \%, 0.5 \%, 0.8 \%$, and $1.3 \%$ of subjects respectively, with no devicerelated death. All 2 cases with major bleeding were attributed to gastrointestinal bleeding. No significant differences were found in the aforementioned complications between the bleeding and non-bleeding groups (Table 2).

\section{Long-term follow-up outcomes}

For 377 patients, a clinical follow up was documented through outpatient service or telephone visits. The average follow-up was 744 days (2.04 years), which yielded 768.5 patient-years in the overall cohort. As shown in Table 3, the average length of follow-up was comparable between the two groups $(P=0.072)$. All the subjects received TEE examination and no difference was presented in TEE examination rate between groups.

Table 3 illustrates the clinical outcomes of an average 2-year follow-up. The incidence of ischemic stroke, TIA, systemic embolism, DRT, intracranial hemorrhage, gastrointestinal bleeding, other major bleedings, $\mathrm{CV} /$ unexplained death, or non-CV / unexplained death in the bleeding group was similar to that in the non-bleeding group, respectively. Additionally, there were no significant differences in major bleeding $(P=0.917)$ and all-cause death $(P=0.313)$ between the two groups. Patients with prior bleeding showed nonsignificant trends toward lower co-primary efficacy events compared to those with no prior bleeding (13.9\% versus $21.7 \%, P=0.063$ ). However, the overall rate of composite thromboembolic events in the cohort was $5.6 \%$, with the bleeding group presenting a significantly lower rate compared with the nonbleeding group throughout the follow-up $(2.2 \%$ versus $7.5 \%, P=0.031)$ (Table 3$)$. After the adjustment of confounders which were chosen based on clinical relevance, such as age, $\geq 75$ years, sex, hypertension, $\mathrm{CHD}$, diabetes, chronic heart failure, previous stroke/TIA, liver dysfunction, and impaired renal function by using propensity score matching (PSM) in the overall cohort, 258 out of 377 patients remained, including 129 in the bleeding group and 129 in the non-bleeding group. Comparison of baseline clinical variables between groups before and after PSM are presented in Table S1 as an online data supplement. KaplanMeier survival curves and a log rank test demonstrated that the cumulative ratio of freedom from allcause death was comparable between the bleeding and non-bleeding groups (HR: $1.065 ; 95 \%$ Cl: 0.6011.889; $\mathrm{P}=0.828$ ) (Figure 2).

Comparisons between the observed annual risk of thromboembolic or bleeding events and the estimated annual risk 
The estimated annual rate of thromboembolic events, based on $\mathrm{CHA}_{2} \mathrm{DS}_{2}-\mathrm{VASc}$ score, was 7.9 per 100 patient-years in the bleeding group and 6.6 per 100 patient-years in the non-bleeding group. However, 3 thromboembolic events (ischemic stroke in 1 patient and TIA in 2 patients) were observed in the bleeding group, and 18 (ischemic stroke in 11 patients and TIA in 7 patients) in the non-bleeding group. Thus, the observed annual rate of thromboembolic events was 1.1 per 100 patient-years, resulting in a relatively risk reduction (RRR) of $86.1 \%\left(\chi^{2}=15.8898, P<0.005\right)$ in the bleeding group, and 3.7 per 100 patient-years, resulting in a RRR of $43.9 \%\left(\chi^{2}=4.3758, P<0.05\right)$ in the non-bleeding group (Figure 3$)$. Meanwhile, the expected annual rate of major bleeding events based on HAS-BLED score was 7.7 per 100 patient-years in the bleeding group and 6.9 per 100 patient-years in the non-bleeding group. In fact, during follow-up, 13 major bleeding events in the bleeding group and 22 in the non-bleeding group were observed. The observed annual rate of bleeding was 4.7 per 100 patient-years and 4.5 per 100 patient-years, which conferred a RRR of $39.0 \%\left(\chi^{2}=3.89, P<0.05\right)$ and a RRR of $34.8 \%\left(\chi^{2}=3.86, P<0.05\right)$ in the bleeding and non-bleeding groups, respectively (Figure 4$)$.

\section{Comparisons of LAAC efficacy in decreasing thromboembolic and bleeding risks between the bleeding and non-bleeding groups}

The patients in the bleeding group had a statistically significantly higher RRR in the annual rate of thromboembolic events compared with those in the non-bleeding group ( $86.1 \%$ vs. $43.9 \%, \chi^{2}=7.03$, $P<0.01)$, whereas no significant difference was found for RRR in the annual rate of major bleeding between the two groups ( $39.0 \%$ vs. $\left.34.8 \%, \chi^{2}=0.29, P>0.05\right)$.

\section{Discussion}

The major findings of our study were as follows. (1) This was one of the larger cohort studies which demonstrated that LAAC is equally safe peri-procedurally between patients with and without prior major bleeding. (2) During long-term follow-up, composite thromboembolic events were lower in patients with previous bleeding than those without bleeding post LAAC procedure; however, major bleeding, all-cause death and co-primary efficacy events were comparable. After PSM, the cumulative ratio of freedom from all-cause death was still similar between groups. (3) LAAC was associated with a lower rate of observed thromboembolic and major bleeding events compared to expected risks based on $\mathrm{CHA}_{2} \mathrm{DS}_{2}$-VASc and HAS-BLED scores in both patients with and without previous major bleeding. (4) Patients with prior major bleeding had a significantly higher RRR in the annual rate of thromboembolic events after LAAC procedure when compared to those without bleeding. (5) LAAC may provide an effective alternative to long-term anticoagulation therapy in patients with NVAF at a higher bleeding risk.

Generally, AF patients with previous bleeding were older and more often had higher $\mathrm{CHA}_{2} \mathrm{DS}_{2}-\mathrm{VASc}$ and HAS-BLED scores than those without previous bleeding [18]. In our study, the bleeding group also exhibited a higher proportion of patients with age $\geq 75$ years old and higher mean $\mathrm{CHA}_{2} \mathrm{DS}_{2}-\mathrm{VASc}$ and HAS-BLED scores. However, the success rate of implantation and rate of severe complications during the 
peri-procedural period were comparable between the two groups. These were in line with conclusions from recent research in which advanced age, higher $\mathrm{CHA}_{2} \mathrm{DS}_{2}$-VASc score or HAS-BLED score, and critically-ill conditions did not seem to influence implantation success, procedure-related adverse events within 7 days, and clinical outcomes [19-21]. In our cohort, the total rate of severe complications in the peri-procedural period within 7 days was $2.9 \%$, with $2.9 \%$ in the bleeding group and $2.9 \%$ in the nonbleeding group, which was lower than the severe adverse events over 7 days of periprocedural period $(4.0 \%)$ in the EWOLUTION registry study [22], and that (6.5\%) reported by Renou et al. in patients with previous intra-cerebral hemorrhage [23] respectively. Therefore, our results reinforced the feasibility and safety of the LAAC strategy regardless of whether the patient had a history of prior major bleeding.

In regard to differences in long-term outcomes between AF patients with and without prior major bleeding, a recent study demonstrated that AF patients with contraindications to OAC had worse thromboembolic and bleeding outcomes at 3 years of follow-up compared with those without contraindications, if no OAC was prescribed [24]. Actually, AF patients with a history of prior bleeding are usually at very high risk of recurrent bleeding when taking OAC. On the other hand, such patients are usually not treated properly with anticoagulation, and are therefore also at high risk for thromboembolic events in clinical practice. Deitelzweig et al. reported that prior baseline bleeding was a strong predictor of not receiving any anticoagulation therapy [25]. For patients with a contraindication to OAC who were on oral anticoagulants, use of OAC could significantly decrease the incidence of mortality, stroke and all-cause hospitalization during long-term follow-up, compared with those not receiving anticoagulation [24]. This study indicated that patients with a high bleeding risk or contraindication to OAC could still benefit from OAC. However, how does a history of prior bleeding affect the long-term outcomes in AF patients who underwent LAAC? Our results showed that composite thromboembolic events were significantly lower in the bleeding group compared to the non-bleeding group at an average 2 years of follow-up post LAAC procedure. Moreover, other endpoints such as major bleeding, all-cause death, and DRT were similar, and the cumulative ratio of freedom from all-cause death was still comparable after adjustment by PSM between patients with or without prior bleeding. These results demonstrated that there was no sufficient evidence to support that a history of prior bleeding was associated with a worse long-term outcome in major bleeding, all-cause mortality and DRT in AF patients after LAAC. On the contrary, we noticed that LAAC could significantly decrease the risk of composite thromboembolic events in patients with versus without previous bleeding. Our results were consistent with the conclusions from recently published literature [26, 27]. In terms of differences in co-primary efficacy events, no significant difference was found between the two groups, but patients with prior major bleeding had a numerically lower trend for co-primary efficacy events with a difference close to statistical significance $(P=0.063)$ when compared to those without prior bleeding. Therefore, it is necessary to further evaluate whether or not patients with prior bleeding can benefit more from LAAC than those without prior bleeding in reducing the risk of coprimary efficacy events in a larger scale trial.

In accordance with our results, López-Mínguez et al. reported that LAAC could decrease the observed annualized rate of stroke and recurrent bleeding events in patients with previous bleeding or ineligible for 
oral anticoagulation, compared with the predicted risks based on $\mathrm{CHA}_{2} \mathrm{DS}_{2}$-VASc and HAS-BLED scores [28]. It was quite evident that patients with prior major bleeding who had higher average $\mathrm{CHA}_{2} \mathrm{DS}_{2}-\mathrm{VASc}$ and HAS-BLED scores in our cohort were at high risk for adverse cardio-cerebrovascular events, but the actual annual rate of thromboembolic and bleeding events was significant lower at 1.1 per 100 patientyears yielding a RRR of $86.1 \%$, and 4.7 per 100 patient-years yielding a RRR of $39.0 \%$ post LAAC procedure, compared to the expected risks respectively. These RRR were identical to those in the EWOLUTION trial which presented a risk reduction of $82.0 \%$ for ischemic stroke/ TIA/ systemic embolism and $30.0 \%$ for recurrent major bleeding in patients with previous major bleeding during 2 years of followup [26]. Similarly, patients without previous bleeding in our study still exhibited a RRR of $43.9 \%$ for thromboembolic events and a RRR of $34.8 \%$ for major bleeding when compared with the predicted risks respectively. Combined with previous studies[29, 30] and our current findings, LAAC seems to be an effective strategy in preventing thromboembolism and decreasing recurrent bleeding risk in AF patients regardless of presence for previous history of hemorrhage.

Regarding the efficacy of LAAC in AF patients who had a prior bleeding, there were several small sample studies which reported the effectiveness and safety of LAAC [31-34]; however, none of them focused on comparing the difference in efficacy of LAAC between patients with and without prior bleeding. Our study compared the extent of RRR in thromboembolic events and major bleeding attributed to LAAC strategy between patients with and without a history of prior bleeding. The results presented that RRR in the annual rate of thromboembolic events was significantly higher in patients with prior bleeding than those with no bleeding; however, RRR in the annual rate of bleeding was not significantly different between groups. Based on these results, it is clear that LAAC not only can decrease the risk of major bleeding events, but also may reduce the risk of thromboembolism in both groups, and more interestingly, LAAC may provide a higher efficacy in depressing risk of thromboembolic events for patients with NVAF and a prior history of bleeding. This implies that the higher the AF patients' risk, the more the patients benefited from the LAAC procedure in preventing thromboembolism. This finding also indicates that the clinical prognosis in lowering risk of thromboembolism is improved more easily in patients with prior bleeding than those without prior bleeding after LAAC intervention.

This study is subject to several limitations. First, this was a retrospective study design conducted in a single center. The major weakness for evaluating the overall efficacy of LAAC is the lack of a randomized control group. Second, inconsistent post-procedure antithrombotic strategies were applied, which might have an impact on clinical outcomes. Third, this cohort study mostly focused on utilization of a single device-WATCHMAN ${ }^{\mathrm{T}}$; thus, the conclusions should not be extrapolated to patients receiving other LAA occluders. Finally, although TEE follow-up was available for all patients, TEE measurement and analysis was conducted only by operators without independent image adjudication.

In conclusion, although AF patients with a history of prior bleeding were at higher risk in thromboembolic events and recurrent bleeding, LAAC provided a safe and effective alternative strategy to reducing thromboembolic and recurrent bleeding risks. During an average 2-year follow-up after LAAC, both thromboembolic and bleeding risks were significantly lower than the predicted rates. Patients with a prior 
major bleeding seemed to benefit more from LAAC in decreasing thromboembolic events compared to those without prior major bleeding. The efficacy of LAAC in reducing thromboembolic risk, rather than lowering major bleeding risk, might be altered by a history of prior bleeding.

\section{Declarations}

\section{Author's contributions}

ZM was a major contributor in collecting, analyzing and interpreting the patient data, as well as writing the manuscript. HCR performed a critical revision for the manuscript. XX performed collection and statistical analysis of data. PF performed data analysis and critical revision. $\mathrm{HN}$ contributed to data analysis. WJ contributed to revision of the manuscript. MZ collected and analyzed the patient data. YJ contributed to study design, analysis of data and critical revision of the manuscript. All authors read and approved the final manuscript.

\section{Funding}

The authors received no specific funding for this work.

\section{Conflict of Interest}

The authors have no conflicts of interest to declare.

\section{Availability of data}

The data supporting this article will be available on reasonable request to the corresponding author.

\section{Consent to participate}

All individual participants gave their informed consent.

\section{Consent for publication}

Not applicable. No identifying information of participants is available in this article.

\section{References}

1. Bai Y, Wang YL, Shantsila A, Lip GYH. The global burden of atrial fibrillation and stroke: a systematic review of the clinical epidemiology of atrial fibrillation in Asia. Chest. 2017; 152: 810-20.

2. 2.Andersson T, Magnuson A, Bryngelsson IL, et al. All-cause mortality in 272,186 patients hospitalized with incident atrial fibrillation 1995-2008: a Swedish nationwide long-term case-control study. Eur Heart J. 2013;34:1061-7.

3. CrestiA, García-Fernández MA, Sievert $\mathrm{H}$, et al. Prevalence of extra-appendage thrombosis in nonvalvular atrial fibrillation and atrial flutter in patients undergoing cardioversion: a large 
transesophageal echo study. Eurolntervention. 2019;15:e225-30.

4. Meersch HVD, Bacquer DD, Vriese ASD. Vitamin $\mathrm{K}$ antagonists for stroke prevention in hemodialysis patients with atrial fibrillation: A systematic review and meta-analysis. Am Heart J. 2017;184:37-46.

5. MartinezC, Katholing A, Wallenhorst C, Freedman Therapy persistence in newly diagnosed nonvalvular atrial fibrillation treated with warfarin or NOAC. A cohort study. Thromb Haemost. 2016 ;115(1):31-9.

6. GrahamDJ, Baro E, Zhang R, et al. Comparative Stroke, Bleeding, and Mortality Risks in Older Medicare Patients Treated with Oral Anticoagulants for Nonvalvular Atrial Fibrillation. Am J Med. $2019 ; 132(5): 596-604$.

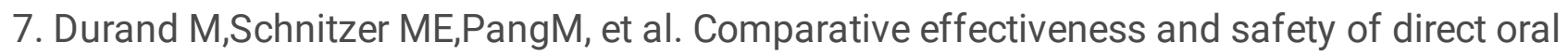
anticoagulants versus vitamin $\mathrm{K}$ antagonists in nonvalvular atrial fibrillation: a Canadian multicentre observational cohort study. CMAJ Open. 2020 ;8(4):E877-86.

8. Bai Y, DengH, ShantsilaA, Lip GYH. Rivaroxaban Versus Dabigatran or Warfarin in Real-World Studies of Stroke Prevention in Atrial Fibrillation: Systematic Review and Meta-Analysis. Stroke. 2017 ;48(4):970-6.

9. Inohara T, Holmes DN, Pieper K, et al. Decline in renal function and oral anticoagulation dose reduction among patients with atrial fibrillation. Heart.2020;106(5):358-64.

10. ReddyVY, Sievert $H$, Halperin J, et al. Percutaneous left atrial appendage closure vs warfarin for atrial fifibrillation. JAMA. 2014;312:1988-98.

11. Holmes DR, Kar S, Price MJ, et al. Prospective randomized evaluation of the Watchman Left Atrial Appendage Closure device in patients with atrial fifibrillation versus long-term warfarin therapy: the PREVAIL trial. J Am Coll Cardiol. 2014;64:1-12.

12. Osmancik P, Herman D, Neuzil P, et al. Left Atrial Appendage Closure Versus Direct Oral Anticoagulants in High-Risk Patients With Atrial Fibrillation. J Am Coll Cardiol. 2020;75(25):3122-35.

13. Mehran R, Rao SV, Bhatt DL, et al. Standardized Bleeding Definitions for Cardiovascular Clinical Trials. A Consensus Report From the Bleeding Academic Research Consortium. Circulation. 2011;123:2736-47.

14. Reddy VY, Holmes D, Doshi SK, Neuzil P, Kar S. Safety of percutaneous left atrial appendage closure: results from the Watchman left atrial appendage system for embolic protection in patients with AF (PROTECT AF) clinical trial and the continued access registry. Circulation. 2011; 123:417-24.

15. Kleinecke $\mathrm{C}$, Yu J, Neef $\mathrm{P}$, et al. Clinical outcomes of Watchman vs. Amplatzer occluders for left atrial appendage closure (WATCH at LAAC). Europace. 2020;22(6):916-23.

16. Friberg L, Rosenqvist M, Lip GY. Evaluation of risk stratification schemes for ischaemic stroke and bleeding in 182678 patients with atrial fibrillation: the Swedish Atrial Fibrillation cohort study. Eur Heart J. 2012;33:1500-10.

17. Lip GY, Frison L, Halperin JL, Lane DA. Comparative validation of a novel risk score for predicting bleeding risk in anticoagulated patients with atrial fibrillation: the HAS-BLED (Hypertension, 
Abnormal Renal/Liver Function, Stroke, Bleeding History or Predisposition, Labile INR, Elderly, Drugs/Alcohol Concomitantly) score. J Am Coll Cardiol. 2011;57:173-80.

18. Garcia DA, Fisher DA, Mulder $\mathrm{H}$, et al. Gastrointestinal bleeding in patients with atrial fibrillation treated with Apixaban or warfarin: Insights from the Apixaban for Reduction in Stroke and Other Thromboembolic Events in Atrial Fibrillation (ARISTOTLE) trial. Am Heart J. 2020 ;221:1-8.

19. Yu J, Chen H, PostF, et al. Efficacy and safety of left atrial appendage closure in non-valvular atrial fibrillation in patients over 75 years. Heart Vessels. 2019; 34(11):1858-65.

20. Chen S, Chun KRJ, Bordignon S, et al. Left atrial appendage occlusion using LAmbre Amulet and Watchman in atrial fibrillation. J Cardiol. $2019 ; 73(4): 299-306$.

21. Brachmann J, Lewalter T, Akin I, et al. Interventional occlusion of left atrial appendage in patients with atrial fibrillation. Acute and long-term outcome of occluder implantation in the LAARGE Registry. J Interv Card Electrophysiol. 2020 ;58(3):273-80.

22. Boersma LVA, Schmidt B, Betts TR, et al. Implant success and safety of left atrial appendage closure with the WATCHMAN device: peri-procedural outcomes from the EWOLUTION registry. Eur Heart J. 2016; 37, 2465-74.

23. RenouP, ThamboJB, Iriart X, et al. Left Atrial Appendage Closure in Patients with Atrial Fibrillation and Previous Intracerebral Hemorrhage. J Stroke Cerebrovasc Dis. 2017 ;26(3):545-51.

24. Steinberg BA, Ballew NG, Greiner MA, et al. Ischemic and Bleeding Outcomes in Patients With Atrial Fibrillation and Contraindications to Oral Anticoagulation. JACC Clin Electrophysiol. 2019 ;5(12):1384-92.

25. Deitelzweig S, Dhamane AD, Fusco MD, et al. Utilization of anticoagulants and predictors of treatment among hospitalized patients with atrial fibrillation in the USA. J Med Econ. 2020; 19:1-12.

26. BoersmaLV, InceH, Kische S, et al. Evaluating Real-World Clinical Outcomes in Atrial Fibrillation Patients Receiving the WATCHMAN Left Atrial Appendage Closure Technology: Final 2-Year Outcome Data of the EWOLUTION Trial Focusing on History of Stroke and Hemorrhage. Circ Arrhythm Electrophysiol. $2019 ; 12(4): \mathrm{e} 006841$.

27. Litwinowicz R, Bartus $M$, Kapelak B, et al. Reduction in risk of stroke and bleedingafter left atrial appendage closurewith LARIAT device in patients with increased risk of stroke and bleeding: Long term results. Catheter Cardiovasc Interv. 2019 ;94(6):837-42.

28. López-Mínguez JR, Nogales-Asensio JM, Infante De Oliveira E, et al. Long-term Event Reduction After Left Atrial Appendage Closure. Results of the Iberian Registry II. Rev Esp Cardiol (Engl Ed).2019 ;72(6):449-55.

29. Regueiro A, Cruz-Gonzalez I, Bethencourt A, et al. Long-term outcomes following percutaneous left atrial appendage closure in patients with atrial fibrillation and contraindications to anticoagulation. $J$ Interv Card Electrophysiol. $2018 ; 52(1): 53-9$.

30. Seeger J, Bothner C, Dahme T, et al. Efficacy and safety of percutaneous left atrial appendage closure to prevent thromboembolic events in atrial fibrillation patients with high stroke and bleeding risk. Clin Res Cardiol.2016;105(3):225-9. 
31. Wintgens LIS, Vorselaars VMM, Klaver MN, et al. Left atrial appendage closure in atrial fibrillation patients with prior major bleeding or ineligible for oral anticoagulation. Neth Heart J. 2019 ;27(12):613-20.

32. HuttE, WazniOM, Saliba WI, et al. Left atrial appendage closure device implantation in patients with prior intracranial hemorrhage. Heart Rhythm. $2019 ; 16(5): 663-8$.

33. Black-Maier E, Piccini JP, Granger CB. Left atrial appendage closure: A therapy uniquely suited for specific populations of patients with atrial fibrillation. J Cardiovasc Electrophysiol. 2019 ;30(12):2968-76.

34. Pouru JP, Lund J, Jaakkola S, et al. Percutaneous left atrial appendage closure in patients with prior intracranial bleeding and Thromboembolism. Heart Rhythm. 2020;17:915-21.

\section{Tables}

Due to technical limitations, table 1 to 3 are only available as a download in the Supplemental Files section.

\section{Figures}




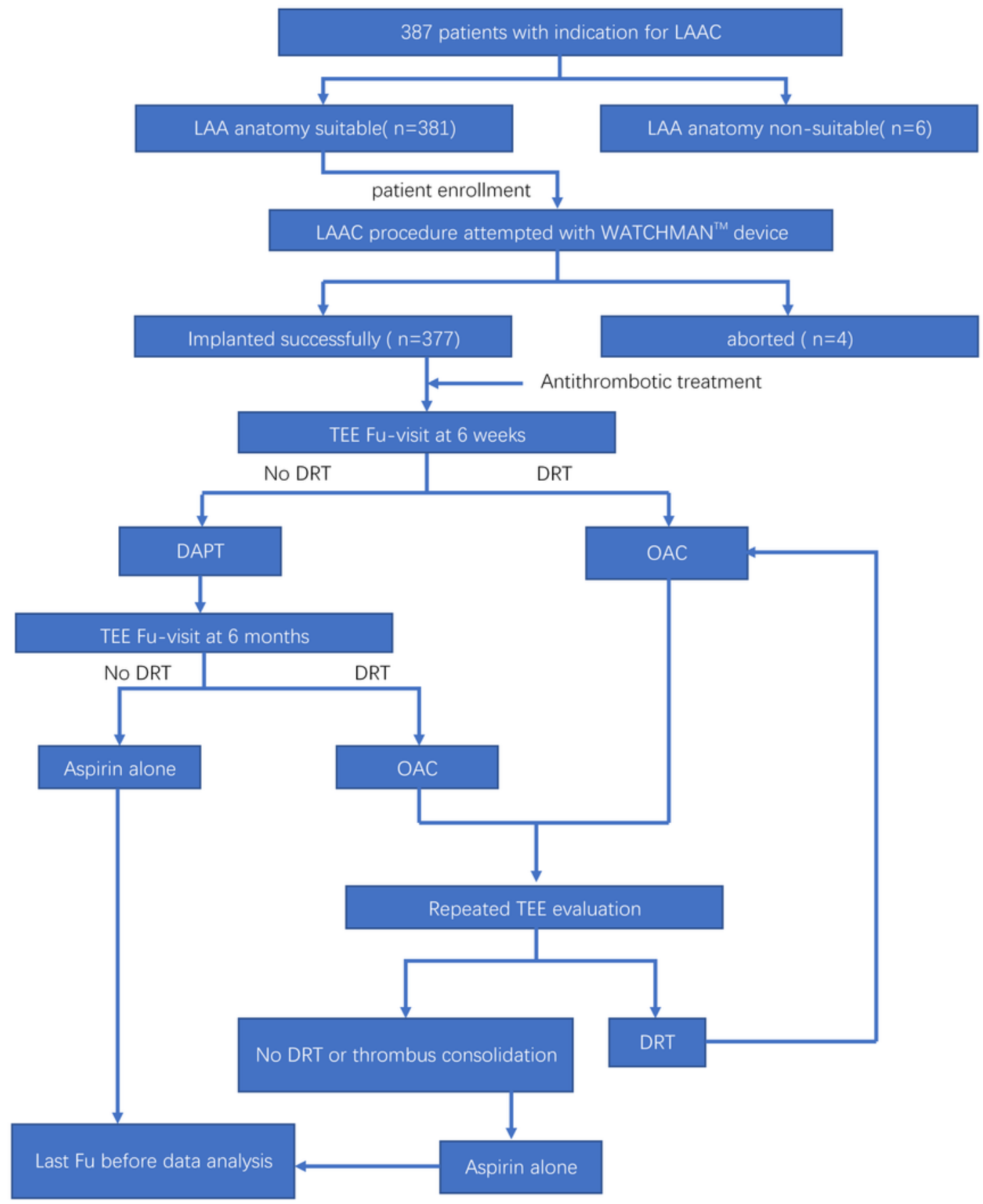

$\mathrm{LAAC}=$ left atrial appendage closere; TEE=transesophageal echocardiography; Fu=follow-up; DRT=device related thrombus; DAPT=dual antiplatelet therapy; $\mathrm{OAC}=$ oral anti-coagulation

\section{Figure 1}

Flowchart of patient inclusion, management and follow-up 


\section{All-cause death}

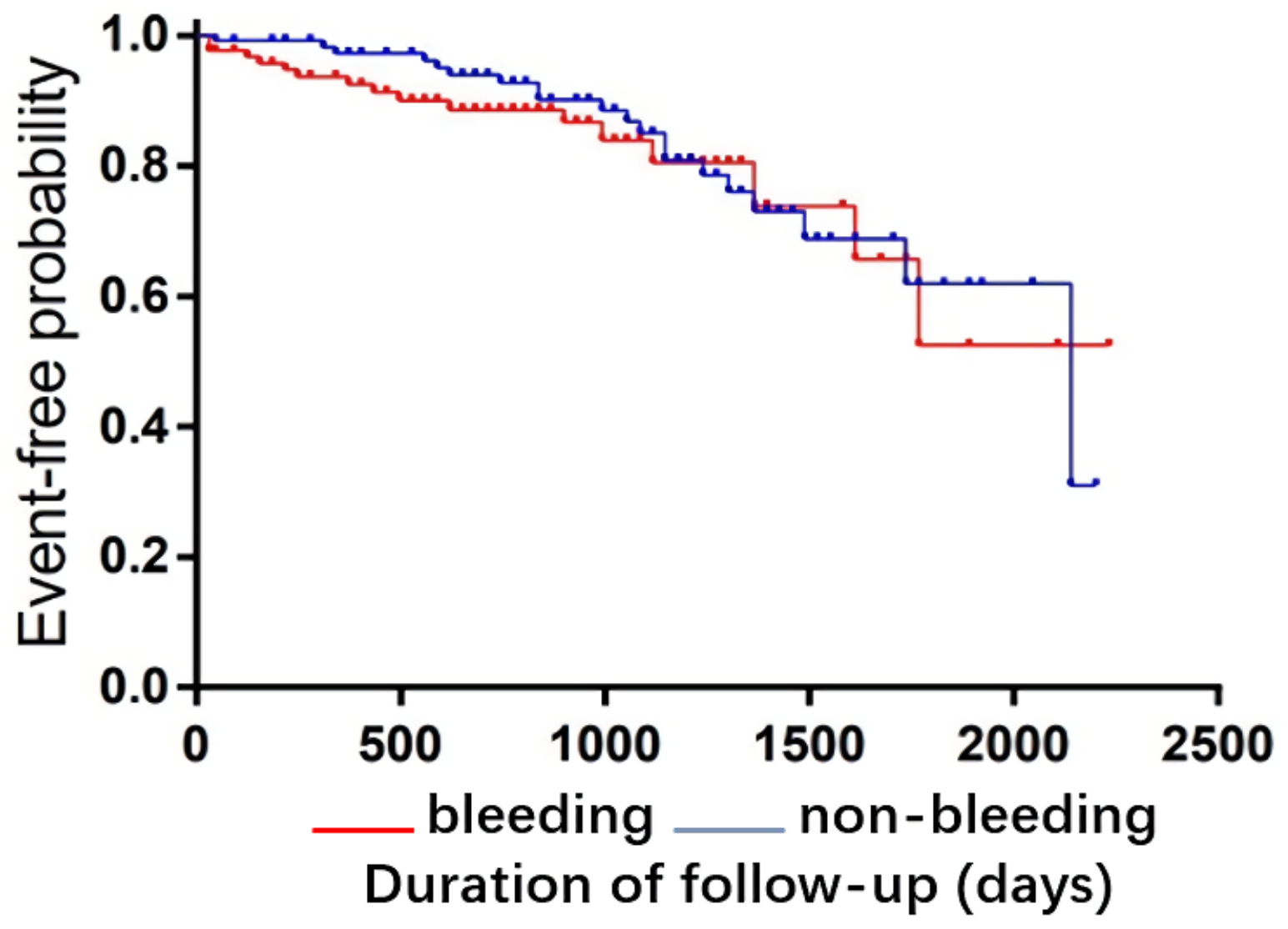

number at risk

$\begin{array}{lcccccc}\text { bleeding } & 137 & 72 & 28 & 10 & 2 & 0 \\ \text { non-bleeding } & 240 & 164 & 98 & 26 & 4 & 0\end{array}$

Figure 2

Kaplan-Meier survival curves of the cumulative ratio of freedom from all-cause death $₫$ bleeding vs. nonbleeding, after adjustment for the potential confounders); Log Rank (Mantel-Cox), HR: 1.065; 95\% Cl: $0.601-1.889 ; P=0.828$ 


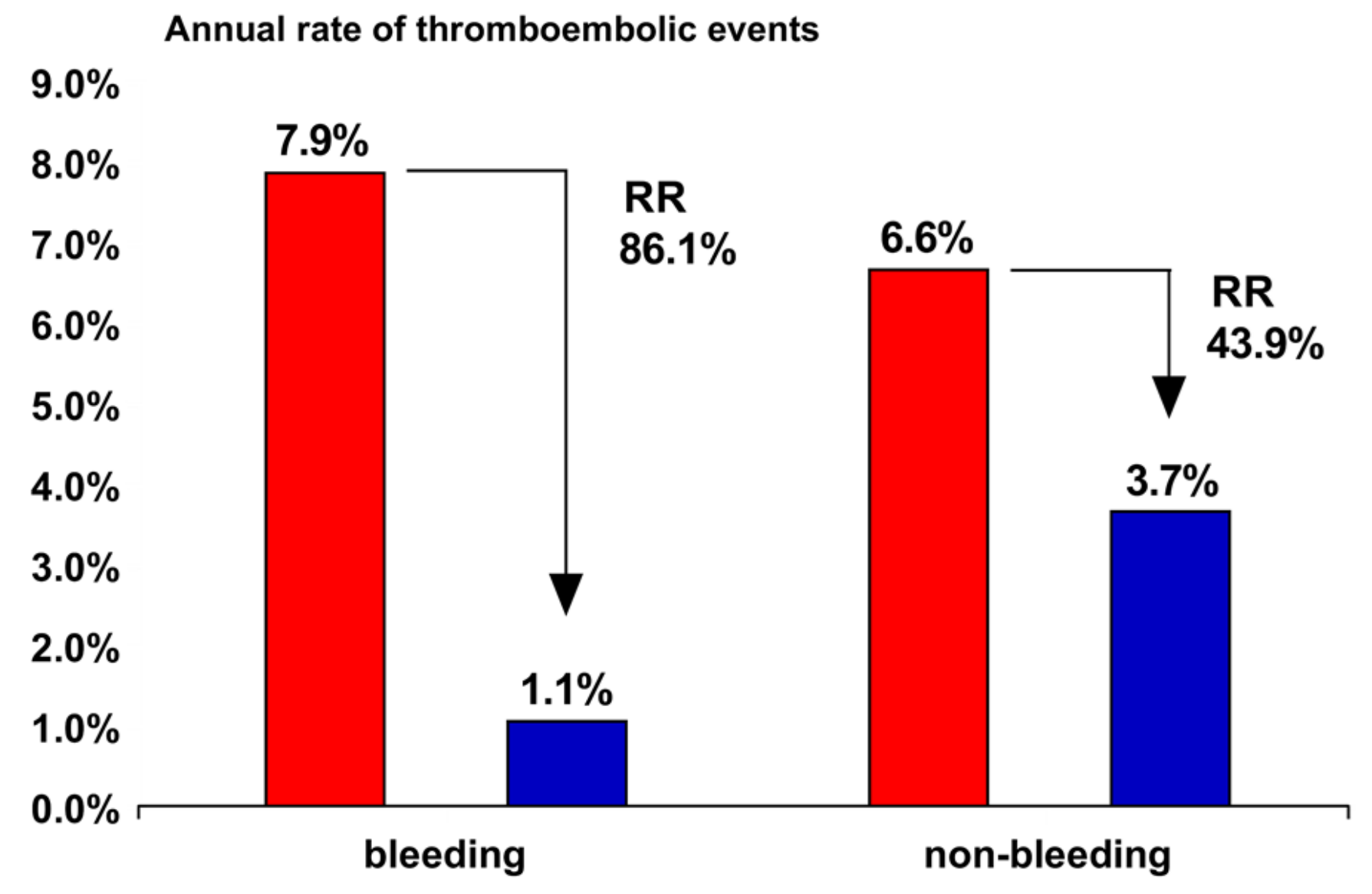

$\square$ Expected, based on $\mathrm{CHA}_{2} \mathrm{DS}_{2}$-VASc $\square$ Observed in our study

Figure 3

Annual rate of composite thromboembolic events: Observed annual rate of composite thromboembolic events, and expected annual rate of composite thromboembolic events based on the CHA2DS2-VASc scores, and the relative risk reduction for bleeding and non-bleeding groups after an average 2 year follow-up. RR囚relative risk 


\section{$9.0 \%$}

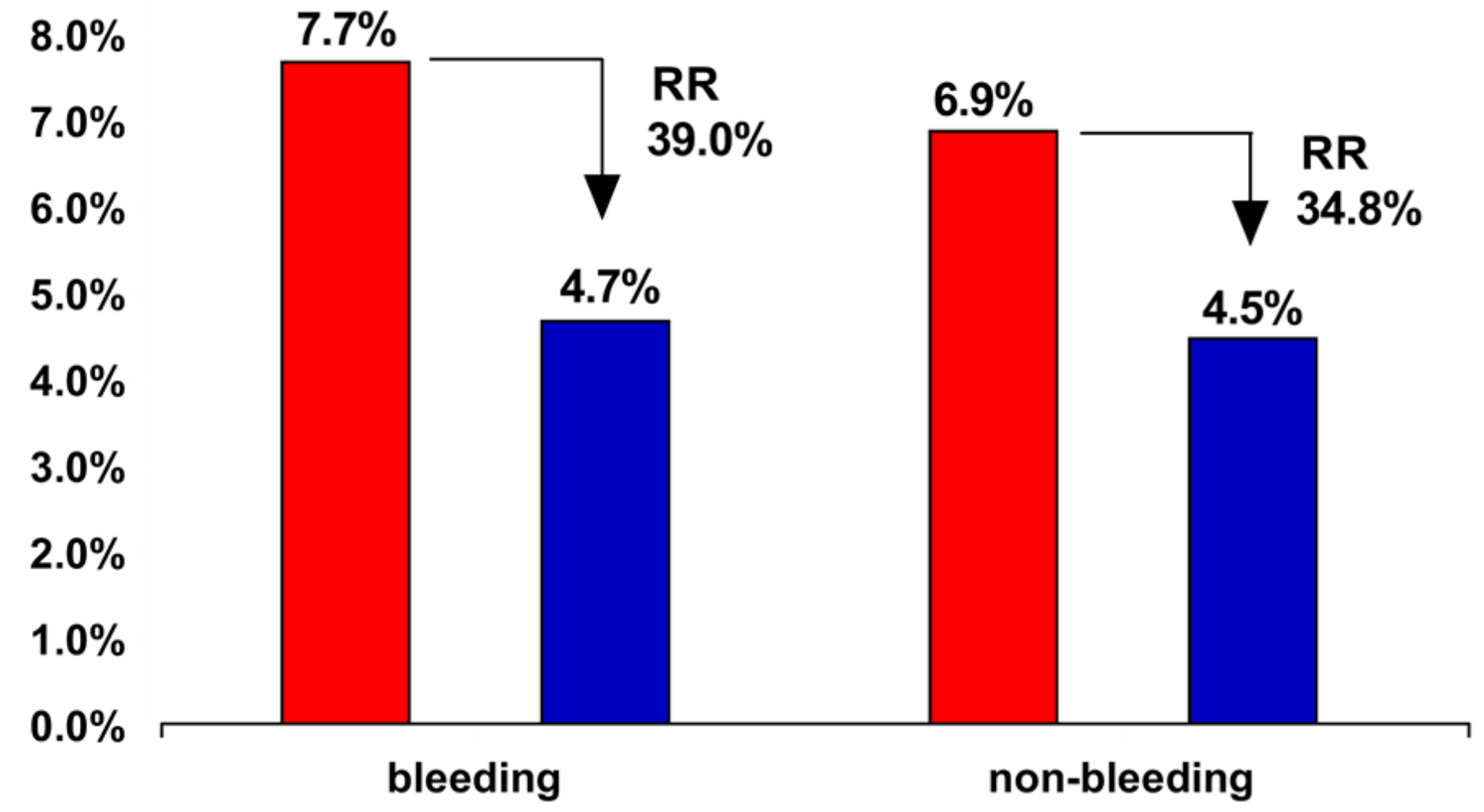

\section{Expected, based on HAS-BLED D Observed in our study}

Figure 4

Annual rate of major bleeding events: Observed annual rate of major bleeding events, and expected annual rate ofmajor bleeding events based on the HAS-BLED score, and the relative risk reduction for bleeding and non-bleeding groups after an average 2 year follow-up. RR囚relative risk

\section{Supplementary Files}

This is a list of supplementary files associated with this preprint. Click to download.

- Supplementarymaterial.doc

- Tables.doc 